\title{
Conservation laws in Skyrme-type models
}

\author{
C. Adam ${ }^{a *}$, J. Sánchez-Guillén ${ }^{a * *}$, and A. Wereszczyński ${ }^{b \dagger}$ \\ ${ }^{a)}$ Departamento de Fisica de Particulas, Universidad de Santiago \\ and Instituto Galego de Fisica de Altas Enerxias (IGFAE) \\ E-15782 Santiago de Compostela, Spain \\ b) Institute of Physics, Jagiellonian University, \\ Reymonta 4, 30-059 Kraków, Poland
}

\begin{abstract}
The zero curvature representation of Zakharov and Shabat has been generalized recently to higher dimensions and has been used to construct non-linear field theories which either are integrable or contain integrable submodels. The Skyrme model, for instance, contains an integrable subsector with infinitely many conserved currents, and the simplest Skyrmion with baryon number one belongs to this subsector. Here we use a related method, based on the geometry of target space, to construct a whole class of theories which are either integrable or contain integrable subsectors (where integrability means the existence of infinitely many conservation laws). These models have three-dimensional target space, like the Skyrme model, and their infinitely many conserved currents turn out to be Noether currents of the volume-preserving diffeomorphisms on target space. Specifically for the Skyrme model, we find both a weak and a strong integrability condition, where the conserved currents form a subset of the algebra of volume-preserving diffeomorphisms in both cases, but this subset is a subalgebra only for the weak integrable submodel.
\end{abstract}

\footnotetext{
*adam@fpaxp1.usc.es

**joaquin@fpaxp1.usc.es

${ }^{\dagger}$ wereszczynski@th.if.uj.edu.pl
} 


\section{Introduction}

Non-linear field theories are important in many fields of physics, with applications ranging from elementary particle theory to condensed matter physics. One feature of these theories which adds to their relevance is the possibility for the existence of extended static (solitons) or stationary (Q-balls) solutions. On the other hand, non-linear field theories are notoriously difficult to analyse, where the degree of difficulty strongly depends on the dimension of the base space (space-time) on which the fields are defined. In $1+1$ dimensions, an ample mathematical apparatus has been developed for the analysis of non-linear theories, among which there are the inverse scattering method, Bäcklund transformations, or the zero curvature representation for integrable systems, which generalizes the Lax pair representation of finite-dimensional integrable systems. Integrability, that is, the existence of infinitely many conserved quantities, is related to all of these methods, and seems to be crucial in the analytical treatment of nonlinear theories, like, e.g., the explicit construction of solutions.

In higher dimensions, much less is known about non-linear field theories. A general concept of integrability has not yet been developed there. One may have, however, theories which contain an integrable subsector like, e.g., in the non-linear sigma model in $2+1$ dimensions, where the integrable subsector is formed by the holomorphic solitons of Belavin and Polyakov. One generalization of the zero curvature representation of Shabat and Zakharov to higher dimensions has been proposed in [1], and it was demonstrated there that this proposal leads to non-linear field theories which have either infinitely many conservation laws in the full theory, or which contain integrable subsectors, defined by some additional constraint equations on the fields, such that the solutions belonging to this subsectors have infinitely many conservation laws. This zero curvature representation, therefore, realizes the concept of integrability in higher-dimensional non-linear field theories in a specific and well-defined manner. These methods also led to the investigation of specific models, and to the analytic construction of both static and time-dependent solutions. For models with infinitely many conservation laws (the so-called AFZ model and related models), static and time-dependent solutions have been constructed, e.g., in [2], 3], 4, [5], and in [6, [7, respectively. Solutions in integrable subsectors of models which are, themselves, not integrable, have been constructed, e.g., in [8], [9]-[11] (the Nicole model and versions thereof) and in [12], [13] (diverse models on base space $S^{3}$ ).

One well-known theory which contains an integrable subsector is the Skyrme model [14, a non-linear field theory with target space $\mathrm{SU}(2)$ (or, equivalently, the three-sphere $S^{3}$ ). In addition, the simplest Skyrmion (i.e., 
the simplest soliton of the Skyrme model with baryon number equal to one) belongs to this integrable subsector, see [15].

The zero curvature representation of Ref. [1] also originated some mathematical applications [16]-[19], in addition to the mentioned construction of infinitely many conserved currents. It is clear that the specific connection one- and two-forms which have been proposed in [1] are not the most general ones, and therefore the approach is not so restrictive. In fact, many applications have shown that most of the new conserved currents in models and their sectors are Noether currents and generalizations thereof, i.e., they are related to the geometry and symmetries of the target space (see [20]). So a direct, geometric approach has been succesfully undertaken to find those currents for models on target space $S^{2}$, corresponding to the Faddeev-Niemi model and modifications of it (like the AFZ and Nicole models), [21, [22]. In [22] it was also found that for models which are not themselves integrable, but contain integrable submodel defined by some constraint, there exist, in fact, weaker constraints (which are easier to obey) which still lead to infinitely many conservation laws. For the sake of clarity we will call the original, stronger constraints (as found, e.g. for the Skyrme model in [15], or for the Faddeev-Niemi model in [23]) the "strong integrability conditions", whereas we will call the weaker constraints which still lead to infinitely many conservtion laws (as found in [22], or in this paper) the "weak integrability conditions".

It is the main aim of this paper to perform the geometric analysis and classification of integrable models and submodels for a general class of Skyrme type models, that is, for theories with the same field content as the Skyrme model (i.e., with three-dimensional target space), analogously to what was done in [21], 22] for general models with two-dimensional target space. We will find that due to the greater complexity of the three-dimensional target space there are significantly more possibilities for integrable models and submodels. Specifically for the Skyrme model we rederive both the original strong integrability condition of [15] and the weaker integrability condition recently found in [24]. Further, we find a class of models with infinitely many target space symmetries for which infinitely many soliton solutions have been constructed recently [24].

The paper is organized as follows. In Section 2 we work out some issues of the Skyrme model which we need in the sequel, especially its properties related to the $S^{3}$ geometry of its target space. These results are known, and some aspects thereof are discussed, e.g., in [25], [26], [27], [28], but we find it useful for our purposes to collect them here and to present them in a geometric formulation. In Section 3 we introduce volume-preserving diffeomorphisms on a three-dimensional manifold and study its properties as well 
as some subsets thereof. Further, we introduce the corresponding Noether currents, when the volume-preserving diffeomorphisms are realized on the target space of a field theory. In Section 4 we introduce a general class of field theories with three-dimensional target space. Then we classify all possibilities for the conservation of all or some of the Noether currents of Section 3 either in the full theory or in a submodel defined by additional integrability conditions. As the number of possibilities to realize this integrability is quite big, we review the results of our classification in three tables at the end of Section 4.

Finally, we want to explain briefly our conventions for partial derivatives. Derivatives w.r.t. target space variables will be denoted frequently by subindices, e.g., $(\partial / \partial a) f \equiv f_{a},(\partial / \partial \xi) f \equiv f_{\xi}$. If an index notation $X^{i}$ is used for target space variables, then the corresponding derivatives are sometimes written like $\left(\partial / \partial X^{i}\right) \equiv \partial_{i}$ or $\left(\partial / \partial X^{3}\right) \equiv \partial_{3}$. Derivatives w.r.t. base space variables (space-time coordinates) are also frequently written as subindices, e.g. $\left(\partial / \partial x^{\mu}\right) u \equiv u_{\mu}, u^{\mu} \equiv \eta^{\mu \nu} u_{\nu}$, when they act on scalar functions (like $u, \xi, a$, etc.). There are some vector-like quantities which carry greek letter (space-time) indices which do not mean derivatives, namely the currents $J_{\mu}$ and the canonical four-momenta $\pi_{\mu}$ and $P_{\mu}$, but there should not be any confusion (greek letter indices on scalar functions always mean derivatives). Observe that also the notation $\left(\partial / \partial u^{\mu}\right) \mathcal{L} \equiv \mathcal{L}_{u^{\mu}}$ does occur.

\section{$2 \quad$ Skyrme model geometry}

The Skyrme model Lagrangian is

$$
\mathcal{L}_{\mathrm{Sk}}=\frac{m^{2}}{2} \mathcal{L}_{2}-\lambda \mathcal{L}_{4}+\frac{M^{4}}{2} \mathcal{L}_{0}
$$

where

$$
\begin{aligned}
\mathcal{L}_{2} & =\operatorname{tr}\left(U^{\dagger} \partial_{\mu} U U^{\dagger} \partial^{\mu} U\right), \\
\mathcal{L}_{4} & =\operatorname{tr}\left[U^{\dagger} \partial_{\mu} U, U^{\dagger} \partial_{\nu} U\right]^{2},
\end{aligned}
$$

and

$$
\mathcal{L}_{0}=\operatorname{tr}(U-\mathbf{1})
$$

where 1 is the $2 \times 2$ unit matrix. Here $m$ and $M$ are constants with the dimension of mass and $\lambda$ is a dimensionless constant. $U$ is a $\mathrm{SU}(2)$-valued matrix field

$$
\begin{aligned}
U: & \mathbb{R} \times \mathbb{R}^{3} \rightarrow \mathrm{SU}(2) \\
& x^{\mu} \rightarrow U\left(x^{\mu}\right)
\end{aligned}
$$


which may be parameterized in the standard manner like

$$
U=e^{i \vec{\xi} \cdot \vec{\sigma}}
$$

Here, $\vec{\sigma} \sim \sigma_{i}, i=1,2,3$ are the Pauli matrices and $\xi_{i}$ are real fields. In the sequel we will use the slightly different parametrization

$$
\xi \equiv|\vec{\xi}| \quad \vec{n} \equiv \frac{\vec{\xi}}{\xi}
$$

such that

$$
U=e^{i \xi \vec{n} \cdot \vec{\sigma}}=\cos \xi 1+i \sin \xi \vec{n} \cdot \vec{\sigma}
$$

and the complex scalar field $u$, which is related to the unit vector field $\vec{n}$ by stereographic projection,

$$
\vec{n}=\frac{1}{(1+u \bar{u})^{2}}(u+\bar{u},-i(u-\bar{u}), 1-u \bar{u}) ; \quad u=\frac{n_{1}+i n_{2}}{1+n_{3}} .
$$

In terms of these variables the Skyrme model Lagrangian is given by

$$
\begin{gathered}
\mathcal{L}_{2}=\xi^{\mu} \xi_{\mu}+4 \sin ^{2} \xi \frac{u^{\mu} \bar{u}_{\mu}}{(1+u \bar{u})^{2}} \\
\mathcal{L}_{4}=16 \sin ^{2} \xi\left(\xi^{\mu} \xi_{\mu} \frac{u^{\nu} \bar{u}_{\nu}}{(1+u \bar{u})^{2}}-\frac{\xi^{\mu} u_{\mu} \xi^{\nu} \bar{u}_{\nu}}{(1+u \bar{u})^{2}}\right)+16 \sin ^{4} \xi \frac{\left(u^{\mu} \bar{u}_{\mu}\right)^{2}-u_{\mu}^{2} \bar{u}_{\nu}^{2}}{(1+u \bar{u})^{4}}
\end{gathered}
$$

and

$$
\mathcal{L}_{0}=\cos \xi-1 \text {. }
$$

The target space $\mathrm{SU}(2)$ is equivalent to $S^{3}$ as a manifold, and the $S^{3}$ geometric aspects of the Skyrme model are especially transparent in the target space coordinates $(\xi, u, \bar{u})$. The metric of $S^{3}$ in these coordinates is

$$
d s^{2}=d \xi^{2}+4 \frac{\sin ^{2} \xi}{(1+u \bar{u})^{2}} d u d \bar{u}
$$

such that the quadratic part of the Skyrme model, $\mathcal{L}_{2}$, is just the pullback under the map $U$ of this metric, whereas the quartic part, $\mathcal{L}_{4}$, is the pullback of an induced metric on area twoforms. Let us be somewhat more precise on this issue. The metric tensor is

$$
\mathbf{g}=d X^{3} \otimes d X^{3}+g\left(d X^{1} \otimes d X^{1}+d X^{2} \otimes d X^{2}\right) \equiv \theta^{1} \otimes \theta^{1}+\theta^{2} \otimes \theta^{2}+\theta^{3} \otimes \theta^{3}
$$

where

$$
X^{3} \equiv \xi \quad X^{1}+i X^{2} \equiv u
$$


are the corresponding real coordinates and $\theta^{i}$ are the co-frame one-forms,

$$
\theta^{3}=d X^{3}, \quad \theta^{1}=\sqrt{g} d X^{1}, \quad \theta^{2}=\sqrt{g} d X^{2}
$$

where

$$
g=4 \frac{\sin ^{2} \xi}{(1+u \bar{u})^{2}}=4 \frac{\sin ^{2} X^{3}}{\left(1+\left(X^{1}\right)^{2}+\left(X^{2}\right)^{2}\right)^{2}}
$$

is the volume density for the metric on $S^{3}$,

$$
g=\operatorname{det}\left(g_{i j}\right), \quad \mathbf{g}=g_{i j} d X^{i} \otimes d X^{j} .
$$

The pullback under the map $U$ of the co-frame one-forms is, e.g., for $\theta^{1}$,

$$
\theta^{1 \prime} \equiv U^{*}\left(\theta^{1}\right)=\sqrt{g} X_{\mu}^{1} d x^{\mu},
$$

and the quadratic part of the Skyrme Lagrangian, $\mathcal{L}_{2}$, is just the sum of the squared norms (length) of these pullbacks in base space (Minkowski space $\left.\mathbb{R}^{3} \times \mathbb{R}\right)$,

$$
\mathcal{L}_{2}=\sum_{i=1}^{3} \tilde{\eta}\left(\theta^{i \prime}, \theta^{i \prime}\right)
$$

where

$$
\begin{aligned}
\sum_{i=1}^{3} \tilde{\eta}\left(\theta^{i \prime}, \theta^{i \prime}\right) & =\left(g\left(X_{\mu}^{1} X_{\nu}^{1}+X_{\mu}^{2} X_{\nu}^{2}\right)+X_{\mu}^{3} X_{\nu}^{3}\right) \tilde{\eta}\left(d x^{\mu}, d x^{\nu}\right) \\
& =\left(\eta^{\mu \nu}\left(g\left(X_{\mu}^{1} X_{\nu}^{1}+X_{\mu}^{2} X_{\nu}^{2}\right)+X_{\mu}^{3} X_{\nu}^{3}\right)\right. \\
& \equiv g\left(\left(X_{\mu}^{1}\right)^{2}+\left(X_{\mu}^{2}\right)^{2}\right)+\left(X_{\mu}^{3}\right)^{2}
\end{aligned}
$$

Here $\tilde{\eta}$ is the metric co-tensor in Minkowski space,

$$
\tilde{\eta}=\eta^{\mu \nu} \partial_{\mu} \otimes \partial_{\nu}
$$

where $\eta^{\mu \nu}=\eta_{\mu \nu}=\operatorname{diag}(1,-1,-1,-1)$. Further, the co-tensor is evaluated on one-forms via the canonical inner product

$$
\partial_{\mu}\left(d x^{\nu}\right)=\delta_{\mu}^{\nu}
$$

as usual.

For an analogous geometric interpretation of the quartic term $\mathcal{L}_{4}$ we introduce the three unit area two-forms

$$
\begin{aligned}
& \Omega_{1}=\theta^{2} \wedge \theta^{3}=\sqrt{g} d X^{2} \wedge d X^{3} \\
& \Omega_{2}=\theta^{3} \wedge \theta^{1}=\sqrt{g} d X^{3} \wedge d X^{1} \\
& \Omega_{3}=\theta^{1} \wedge \theta^{2}=g d X^{1} \wedge d X^{2}
\end{aligned}
$$


Their pull-backs under the map $U$ are, e.g., for $\Omega_{3}$,

$$
\Omega_{3}{ }^{\prime} \equiv U^{*}\left(\Omega_{3}\right)=g X_{\mu}^{1} X_{\nu}^{2} d x^{\mu} \wedge d x^{\nu}
$$

and the quartic part $\mathcal{L}_{4}$ is equal to the sum of the squared lengths of these three pullbacks,

$$
\mathcal{L}_{4}=\sum_{i=1}^{3}\left|\Omega_{i}^{\prime}\right|^{2}
$$

where, e.g.,

$$
\begin{aligned}
\left|\Omega_{3}{ }^{\prime}\right|^{2} & =g^{2} X_{\mu}^{1} X_{\nu}^{2} X_{\alpha}^{1} X_{\beta}^{2}\left[\tilde{\eta}\left(d x^{\mu}, d x^{\alpha}\right) \tilde{\eta}\left(d x^{\nu}, d x^{\beta}\right)-\tilde{\eta}\left(d x^{\mu}, d x^{\beta}\right) \tilde{\eta}\left(d x^{\nu}, d x^{\alpha}\right)\right] \\
& =g^{2}\left(X_{\mu}^{1} X_{\nu}^{2} X^{1 \mu} X^{2 \nu}-X_{\mu}^{1} X_{\nu}^{2} X^{1 \nu} X^{2 \mu}\right) \\
& \equiv g^{2}\left[\left(X_{\mu}^{1}\right)^{2}\left(X_{\nu}^{2}\right)^{2}-\left(X_{\mu}^{1} X^{2 \mu}\right)^{2}\right]
\end{aligned}
$$

The potential term (or pion mass term) $\mathcal{L}_{0}$ does not have a similar interpretation in terms of the $S^{3}$ geometry of the target space. This term is, however, sometimes omitted, and we shall treat both the case with and without this term.

The geometric structure of the Skyrme model without the potential term $\mathcal{L}_{0}$ is also reflected in its target space symmetries. Indeed, both the quadratic and the quartic term are invariant under the transformation

$$
U \rightarrow V U W^{\dagger}, \quad V, W \in \mathrm{SU}(2) .
$$

(The pion mass term is only invariant under the diagonal subgroup $V=W$.) The transformation on $U$ is the same for $(V, W)$ and $(-V,-W)$, therefore the target space symmetry group is $\mathrm{SU}(2) \times \mathrm{SU}(2) / \mathbb{Z}_{2} \sim \mathrm{SO}(4)$. Further, $S O(4)$ is the isometry group of the three-sphere $S^{3}$, so the above geometric identification of the Skyrme Lagrangian leads to the assumption that the target space symmetries of the Skyrme model (without pion mass term) are just the isometries of the $S^{3}$ target space metric. And this is indeed the case. For infinitesimal transformations

$$
V=1+i \vec{\alpha} \cdot \vec{\sigma}, \quad W=1+i \vec{\beta} \cdot \vec{\sigma}
$$

and using the parametrization in terms of $u, \bar{u}, \xi$ for $U$, the action of these infinitesimal transformations on $u, \bar{u}, \xi$ may be calculated to be $u \rightarrow u+Y^{u}$, $\xi \rightarrow \xi+Y^{\xi}$ with

$$
Y^{u}=\frac{1}{2}\left(\frac{\cos \xi}{\sin \xi}\left(\gamma_{+}-u^{2} \gamma_{-}-2 u \gamma_{3}\right)+i\left(\delta_{+}-u^{2} \delta_{-}-2 u \delta_{3}\right)\right)
$$




$$
Y^{\xi}=\frac{1}{1+u \bar{u}}\left[\bar{u} \gamma_{+}+u \gamma_{-}+(1-u \bar{u}) \gamma_{3}\right]
$$

where

$$
\vec{\gamma} \equiv \vec{\alpha}-\vec{\beta}, \quad \vec{\delta} \equiv \vec{\alpha}+\vec{\beta}, \quad \gamma_{ \pm} \equiv \gamma_{1} \pm i \gamma_{2}, \quad \delta_{ \pm} \equiv \delta_{1} \pm i \delta_{2}
$$

and $\vec{\delta}$ parametrizes the diagonal subgroup $V=W$. The six corresponding vector fields into the directions $\vec{\gamma}$ and $\vec{\delta}$ indeed span the Lie algebra of $\mathrm{SO}(4)$ and leave invariant the metric (13) on $S^{3}$ (i.e., the obey the Killing equation), so they generate the isometries of the target space of the Skyrme model.

These symmetries define six conserved Noether currents in the Skyrme model. There exist further Noether currents in the model which are not conserved in the full model. They are, however, conserved in a submodel where the fields $u, \bar{u}, \xi$ obey, in addition to the field equations, the constraints

$$
\left(\partial_{\mu} u\right)^{2}=0, \quad \partial^{\mu} \xi \partial_{\mu} u=0 .
$$

Then, one can construct two classes of infinitely many conserved currents [15], namely

$$
J_{\mu}^{G}=i g^{-1}\left(G_{u} \bar{\pi}_{\mu}-G_{\bar{u}} \pi_{\mu}\right)
$$

and

$$
J_{\mu}^{\left(H^{(1)}, H^{(2)}\right)}=(1+u \bar{u})^{2}\left[2 \frac{\cos \xi}{\sin \xi}\left(H^{(1)} \bar{\pi}_{\mu}+H^{(2)} \pi_{\mu}\right)-\left(H_{\bar{u}}^{(1)}+H_{u}^{(2)}\right) P_{\mu}\right]
$$

where $G$ is an arbitrary function of $\xi, u, \bar{u}$ whereas $H^{(1)}, H^{(2)}$ depend only on $u$ and $\bar{u}$. Further,

$$
\pi_{\mu} \equiv \mathcal{L}_{u^{\mu}}, \quad P_{\mu} \equiv \mathcal{L}_{\xi^{\mu}}
$$

are the canonical four-momenta of the Skyrme model. The second class of currents is not real for $H^{(1)} \neq H^{(2)}$ but an equivalent set of real currents is easily found to be

$$
\begin{array}{r}
J_{\mu}^{\left(H^{(1)}\right)}=(1+u \bar{u})^{2}\left[2 \frac{\cos \xi}{\sin \xi} H^{(1)}\left(\bar{\pi}_{\mu}+\pi_{\mu}\right)-\left(H_{\bar{u}}^{(1)}+H_{u}^{(1)}\right) P_{\mu}\right] \\
J_{\mu}^{\left(H^{(2)}\right)}=i(1+u \bar{u})^{2}\left[2 \frac{\cos \xi}{\sin \xi} H^{(2)}\left(\bar{\pi}_{\mu}-\pi_{\mu}\right)-\left(H_{\bar{u}}^{(2)}-H_{u}^{(2)}\right) P_{\mu}\right] .
\end{array}
$$

We shall see in the next section that these currents form, in fact, a subset of the Noether currents of the volume-preserving diffeomorphisms on the target space of the Skyrme model.

Remark 1: the Noether currents of the target space symmetries form, of course, a subset of the above Noether currents. More precisely, the Noether 
currents of the diagonal subgroup $(\vec{\gamma}=0)$ are of the type (34), whereas the other three Noether currents $(\vec{\delta}=0)$ are of the type (37), (38).

Remark 2: we will see in the next section that the charges of the Noether currents of the type (37), (38) do not close as a Lie algebra, neither among themselves, nor together with the charges of the currents (34), whereas the charges of the currents (34) do close as a Lie algebra among themselves. There exist, however, subsets of the currents (34), (37) and (38) which do form a closed Lie algebra, like, e.g., the six generators of the target space symmetry group $\mathrm{SO}(4)$ (see Remark 1 above).

\section{$3 \quad$ Volume preserving diffeomorphisms}

The volume $n$-form on an $n$ dimensional manifold $\mathcal{M}^{n}$ is

$$
d V=g\left(X^{i}\right) d X^{1} \wedge d X^{2} \wedge \ldots d X^{n} \quad, \quad i=1, \ldots n
$$

where we use capital letters $X^{i}$ for the coordinates, because the manifold will be identified with target space later on. Here $g$ is the volume density. If $\mathcal{M}^{n}$ is a Riemannian manifold with metric $g_{i j}$, then the volume density $g$ is the square-root of the determinant of the metric $g_{i j}$. A volume-form preserving diffeomorphism is a coordinate transformation $X^{i} \rightarrow X^{i}\left(X^{i}\right)$ such that the volume form remains invariant, $g\left(X^{\prime i}\right) d X^{\prime 1} \ldots d X^{\prime n}=g\left(X^{i}\right) d X^{1} \ldots d X^{n}$. For an infinitesimal transformation

$$
X^{i}=X^{i}+\epsilon Y^{i}\left(X^{j}\right)
$$

invariance of the volume form requires

$$
\partial_{i}\left(g Y^{i}\right) \equiv \frac{\partial}{\partial X^{i}}\left(g Y^{i}\right)=0 .
$$

If the manifold (target space) is three-dimensional, $n=3$, then a general (local) solution to this equation is provided by Darboux's theorem,

$$
Z^{i} \equiv g Y^{i}=\epsilon^{i j k} \partial_{j} A \partial_{k} B
$$

where $A, B$ are arbitrary functions of the $X^{i}$. The functions $A, B$ are called Clebsch variables, and their choice is not unique (that is, different $A, B$ can give rise to the same $Y^{i}$ ). Therefore, a general vector field generating a volume preserving diffeomorphism reads

$$
\mathbf{v}^{(Y)}=Y^{i} \partial_{i}=h \epsilon^{i j k}\left(\partial_{j} A\right)\left(\partial_{k} B\right) \partial_{i} \quad, \quad h \equiv g^{-1} .
$$


For later convenience we introduce the vector field components corresponding to the target space coordinates (15) of Section 2,

$$
Y^{u}=Y^{1}+i Y^{2} \quad Y^{\bar{u}}=Y^{1}-i Y^{2}, \quad Y^{\xi}=Y^{3},
$$

such that the above vector field is re-expressed like

$$
\begin{aligned}
\mathbf{v}^{(Y)}= & Y^{u} \partial_{u}+Y^{\bar{u}} \partial_{\bar{u}}+Y^{\xi} \partial_{\xi} \\
= & 2 i h\left[\left(A_{\xi} B_{\bar{u}}-A_{\bar{u}} B_{\xi}\right) \partial_{u}-\left(A_{\xi} B_{u}-A_{u} B_{\xi}\right) \partial_{\bar{u}}\right. \\
& \left.-\left(A_{u} B_{\bar{u}}-A_{\bar{u}} B_{u}\right) \partial_{\xi}\right]
\end{aligned}
$$

where now $A$ and $B$ are functions of $u, \bar{u}, \xi$.

The algebra of volume-preserving vector fields closes,

$$
\begin{gathered}
{\left[\mathbf{v}^{(Y)}, \mathbf{v}^{(\tilde{Y})}\right]=\mathbf{v}^{(\tilde{Y})}} \\
\tilde{\tilde{Y}}^{i}=\left(\partial_{j} Y^{i}\right) \tilde{Y}^{j}-\left(\partial_{j} \tilde{Y}^{i}\right) Y^{j}, \quad \partial_{i}\left(g \tilde{\tilde{Y}}^{i}\right)=0 .
\end{gathered}
$$

Next, we want to investigate a subset of the volume-preserving diffeomorphisms obeying an additional condition, because we will find in the next section that for many models the corresponding Noether currents are conserved only for this subset (see Eq. (96)). Concretely, the condition defining the subset is

$$
\partial_{3} Y^{3}=0 .
$$

This set does not form a subalgebra, as may be checked easily. Indeed, for $\partial_{3} Y^{3}=\partial_{3} \tilde{Y}^{3}=0$ we find

$$
\partial_{3} \tilde{\tilde{Y}}^{3}=\left(\partial_{1} Y^{3}\right)\left(\partial_{3} \tilde{Y}^{1}\right)+\left(\partial_{2} Y^{3}\right)\left(\partial_{3} \tilde{Y}^{2}\right)-\left(\partial_{1} \tilde{Y}^{3}\right)\left(\partial_{3} Y^{1}\right)-\left(\partial_{2} \tilde{Y}^{3}\right)\left(\partial_{3} Y^{2}\right)
$$

which is nonzero in general. There exist, however, closed subalgebras within this set. One subalgebra is defined by the condition that

$$
Y^{3} \equiv 0
$$

because it follows immediately from Eq. (47) that $Y^{3}=0 \wedge \tilde{Y}^{3}=0 \Rightarrow$ $\tilde{\tilde{Y}}^{3}=0$. In terms of the functions $A$ and $B$ there are different possibilities to fulfill $Y^{3}=0$. One may e.g., choose

$$
B=B\left(X^{3}\right) \quad \Rightarrow \partial_{1} B=\partial_{2} B=0
$$

which leads to

$$
Y^{1}=h\left(\partial_{2} A\right)\left(\partial_{3} B\right), \quad Y^{2}=-h\left(\partial_{1} A\right)\left(\partial_{3} B\right), \quad Y^{3}=0 .
$$


The same $Y^{i}$ are obtained when the transformation $A \rightarrow\left(\partial_{3} B\right) A, B \rightarrow X^{3}$ is performed, therefore a general vector field $Y^{i}$ of this type is given by

$$
Y^{1}=h\left(\partial_{2} A\right), \quad Y^{2}=-h\left(\partial_{1} A\right), \quad Y^{3}=0 .
$$

Observe that these are precisely the vector fields leading to the Noether currents (34) of Section 2, once the identification $A \rightarrow G$ is made. Therefore, the geometric method of this section indeed provides an alternative way to find the Noether currents which have been originally derived from the generalized curvature representation.

An abelian subalgebra of this algebra is obtained by restricting $A$ to

$$
A=A\left(a, X^{3}\right), \quad B=X^{3}, \quad a=\left(X^{1}\right)^{2}+\left(X^{2}\right)^{2}=u \bar{u}
$$

in which case

$$
Y^{1}=2 X^{2} h A_{a} \quad, \quad Y^{2}=-2 X^{1} h A_{a} .
$$

An apparently different choice with $Y^{3}=0$ is $A=A\left(a, X^{3}\right), B=B\left(a, X^{3}\right)$, but this leads to $\left(\xi \equiv X^{3}, B_{\xi} \equiv \partial_{\xi} B\right)$

$$
Y^{1}=2 X^{2} h\left(A_{a} B_{\xi}-A_{\xi} B_{a}\right) \quad, \quad Y^{2}=-2 X^{1} h\left(A_{a} B_{\xi}-A_{\xi} B_{a}\right)
$$

and therefore to the same abelian subalgebra (both $Y^{1}$ and $Y^{2}$ depending on one single function of $a$ and $\xi$ ).

There seems to exist another subalgebra, namely $\partial_{3} Y^{i}=0$ for all three components of $Y$, which indeed implies $\partial_{3} \tilde{\tilde{Y}}^{i}=0$, see Eq. (47). However, we have not been able to find a nontrivial solution to this condition for nontrivial $g$ (i.e., $g_{\xi} \neq 0$ ).

Another set of vector fields obeying $\partial_{3} Y^{3}=0$ and $\partial_{i}\left(g Y^{i}\right)=0$ can be found for special factorising $g$ of the form

$$
g=g^{(1)}\left(X^{1}, X^{2}\right) g^{(2)}\left(X^{3}\right) .
$$

This set is given by

$$
\begin{gathered}
Y^{1}=g^{-1}\left(\partial_{3} g^{(2)}\right) H^{(1)}, \quad Y^{2}=(g)^{-1}\left(\partial_{3} g^{(2)}\right) H^{(2)}, \\
Y^{3}=-\left(g^{(1)}\right)^{-1}\left(\partial_{1} H^{(1)}+\partial_{2} H^{(2)}\right)
\end{gathered}
$$

where $H^{(1)}=H^{(1)}\left(X^{1}, X^{2}\right)$ and $H^{(2)}=H^{(2)}\left(X^{1}, X^{2}\right)$ are arbitrary functions of $X^{1}$ and $X^{2}$ only. These vector fields lead precisely to the Noether currents (37) and (38) of Section 2.

In the special case of $H^{(2)}=0$ the corresponding Clebsch variables are

$$
A=\sqrt{2 g^{(2)} H^{(1)}} \cos X^{2}, \quad B=-\sqrt{2 g^{(2)} H^{(1)}} \sin X^{2}
$$


and for $H^{(1)}=0$ they are

$$
A=\sqrt{2 g^{(2)} H^{(1)}} \cos X^{1}, \quad B=\sqrt{2 g^{(2)} H^{(1)}} \sin X^{1}
$$

whereas they are more complicated in the general case. This set of vector fields does not form a subalgebra, however, for general $H^{(1)}$ and $H^{(2)}$ (i.e. $\partial_{3} \tilde{\tilde{Y}}^{3}=0$ does not hold in general). It does form a subalgebra for special choices of $H^{(1)}, H^{(2)}$, like, e.g., $H^{(1)}=H^{(1)}\left(X^{2}\right)$ and $H^{(2)}=H^{(2)}\left(X^{1}\right)$, or for $H^{(1)}=\partial_{2} H$ and $H^{(2)}=-\partial_{1} H$. But in these special cases $Y^{3}=0$ and, therefore, they are included in the subalgebra discussed above.

Finally, we give the general expression for Noether currents in a relativistic field theory which correspond to the vector fields $\mathbf{v}^{(Y)}$ that generate volume preserving diffeomorphisms on target space. They are

$$
J_{\mu}^{(Y)}=Y^{u} \pi_{\mu}+Y^{\bar{u}} \bar{\pi}_{\mu}+Y^{\xi} P_{\mu}
$$

where $\pi_{\mu}=\partial_{u^{\mu}} \mathcal{L}$ and $P_{\mu}=\partial_{\xi^{\mu}} \mathcal{L}$ are the usual canonical four-momenta, and $u^{\mu} \equiv \eta^{\mu \nu} \partial_{\nu} u$, etc. ( $\eta^{\mu \nu}$ is the space-time metric). The charges $Q^{(Y)}=$ $\int d^{3} \mathbf{r} J_{0}^{(Y)}$ generate the algebra of the vector fields $\mathbf{v}^{(Y)}$ under the Poisson bracket

$$
\left\{u\left(\mathbf{r}_{1}\right), \pi_{0}\left(\mathbf{r}_{2}\right)\right\}=\delta\left(\mathbf{r}_{1}-\mathbf{r}_{2}\right), \quad\left\{\xi\left(\mathbf{r}_{1}\right), P_{0}\left(\mathbf{r}_{2}\right)\right\}=\delta\left(\mathbf{r}_{1}-\mathbf{r}_{2}\right)
$$

as usual.

\section{Conserved currents in Skyrme type models}

We shall now perform the analysis of conservation laws along the lines of what was done for Faddeev-Niemi and related models in [21, [22], investigating the possibilities of conserved currents for Skyrme and related models, with special attention to the integrability conditions and the sectors they define. First, let us introduce the abbreviations

$$
\begin{gathered}
a=u \bar{u}, \quad b=u^{\mu} \bar{u}_{\mu}, \quad c=\left(u^{\mu} \bar{u}_{\mu}\right)^{2}-u_{\mu}^{2} \bar{u}_{\nu}^{2} \\
d=\xi^{\mu} \xi_{\mu}, \quad e=\xi^{\mu} u_{\mu} \xi^{\nu} \bar{u}_{\nu}
\end{gathered}
$$

such that the quadratic and the quartic part of the Skyrme Lagrangian may be re-expressed like

$$
\mathcal{L}_{2}=d+4 \frac{\sin ^{2} \xi}{(1+a)^{2}} b
$$


and

$$
\mathcal{L}_{4}=\frac{\sin ^{2} \xi}{(1+a)^{2}}(b d-e)+\frac{\sin ^{4} \xi}{(1+a)^{4}} c .
$$

Further, we will also study more general Lagrangians $\mathcal{L}=\mathcal{L}(a, b, c, \xi, d, e)$ with the following canonical four-momenta

$$
\begin{gathered}
\pi_{\mu}=\mathcal{L}_{u^{\mu}}=\bar{u}_{\mu} \mathcal{L}_{b}+2\left(b \bar{u}_{\mu}-\bar{u}_{\nu}^{2} u_{\mu}\right) \mathcal{L}_{c}+\left(\xi^{\nu} \bar{u}_{\nu}\right) \xi_{\mu} \mathcal{L}_{e} \\
P_{\mu}=\mathcal{L}_{\xi^{\mu}}=2 \xi_{\mu} \mathcal{L}_{d}+\left(\left(\xi^{\nu} \bar{u}_{\nu}\right) u_{\mu}+\left(\xi^{\nu} u_{\nu}\right) \bar{u}_{\mu}\right) \mathcal{L}_{e}
\end{gathered}
$$

field equations

$$
\partial^{\mu} \pi_{\mu}=\mathcal{L}_{u}=\bar{u} \mathcal{L}_{a}, \quad \partial^{\mu} P_{\mu}=\mathcal{L}_{\xi}
$$

and the following useful identities

$$
\begin{aligned}
u^{\mu} \pi_{\mu} & =b \mathcal{L}_{b}+2 c \mathcal{L}_{c}+e \mathcal{L}_{e} \\
\bar{u}^{\mu} \pi_{\mu} & =\bar{u}_{\mu}^{2} \mathcal{L}_{b}+\left(\bar{u}_{\mu} \xi^{\mu}\right)^{2} \mathcal{L}_{e} \\
\xi^{\mu} \pi_{\mu} & =\left(\xi^{\mu} \bar{u}_{\mu}\right) \mathcal{L}_{b}+2\left(b \xi^{\mu} \bar{u}_{\mu}-\bar{u}_{\nu}^{2} \xi^{\mu} u_{\mu}\right) \mathcal{L}_{c}+d \xi^{\mu} \bar{u}_{\mu} \mathcal{L}_{e} \\
u^{\mu} P_{\mu} & =2\left(\xi^{\mu} u_{\mu}\right) \mathcal{L}_{d}+\left(\left(\xi^{\mu} \bar{u}_{\mu}\right) u_{\nu}^{2}+b \xi^{\mu} u_{\mu}\right) \mathcal{L}_{e} \\
\xi^{\mu} P_{\mu} & =2 d \mathcal{L}_{d}+2 e \mathcal{L}_{e} .
\end{aligned}
$$

Now we want to calculate the divergence of the Noether currents (61)

$$
\begin{aligned}
\partial^{\mu} J_{\mu}^{(Y)=} & \left(Y_{u}^{u} u^{\mu}+Y_{\bar{u}}^{u} \bar{u}^{\mu}+Y_{\xi}^{u} \xi^{\mu}\right) \pi_{\mu}+\left(Y_{u}^{\bar{u}} u^{\mu}+Y_{\bar{u}}^{\bar{u}} \bar{u}^{\mu}+Y_{\xi}^{\bar{u}} \xi^{\mu}\right) \bar{\pi}_{\mu}+ \\
& \left(Y_{u}^{\xi} u^{\mu}+Y_{\bar{u}}^{\xi} \bar{u}^{\mu}+Y_{\xi}^{\xi} \xi^{\mu}\right) P_{\mu}+Y^{u} \partial^{\mu} \pi_{\mu}+Y^{\bar{u}} \partial^{\mu} \bar{\pi}_{\mu}+Y^{\xi} \partial^{\mu} P_{\mu} .
\end{aligned}
$$

In a first step we want to restrict to the strong integrability conditions

$$
u^{\mu} \xi_{\mu}=0 \quad, \quad u_{\mu}^{2}=0
$$

These are the integrability conditions which follow from the generalized zero curvature representation for the Skyrme model and lead to the infinitely many conserved currents (34), (37) and (38) in this case, see [15]. The strong integrability conditions imply

$$
u^{\mu} \bar{\pi}_{\mu}=0, \quad u^{\mu} P_{\mu}=0, \quad \xi^{\mu} \pi_{\mu}=0,
$$

and we find for the current divergence

$$
\left.\partial^{\mu} J_{\mu}^{(Y)}\right|_{\mathrm{s}}=\left(Y_{u}^{u}+Y_{\bar{u}}^{\bar{u}}\right) u^{\mu} \pi_{\mu}+Y^{u} \mathcal{L}_{u}+Y^{\bar{u}} \mathcal{L}_{\bar{u}}+Y_{\xi}^{\xi} \xi^{\mu} P_{\mu}+Y^{\xi} \mathcal{L}_{\xi},
$$


where we used $u^{\mu} \pi_{\mu}=\bar{u}^{\mu} \bar{\pi}_{\mu}$. Next we express $Y^{i}$ like

$$
Y^{i}=g^{-1} Z^{i} \equiv h Z^{i} \quad \Rightarrow \quad \partial_{i} Z^{i}=0
$$

and get

$$
\begin{aligned}
\left.\partial^{\mu} J_{\mu}^{(Y)}\right|_{\mathrm{s}}= & Z^{u}\left(h_{u} u^{\mu} \pi_{\mu}+h \mathcal{L}_{u}\right)+Z^{\bar{u}}\left(h_{\bar{u}} u^{\mu} \pi_{\mu}+h \mathcal{L}_{\bar{u}}\right)+ \\
& Z^{\xi}\left(h_{\xi} u^{\mu} \pi_{\mu}+h \mathcal{L}_{\xi}\right)+\left(h Z^{\xi}\right)_{\xi}\left(\xi^{\mu} P_{\mu}-u^{\mu} \pi_{\mu}\right) .
\end{aligned}
$$

Now we assume that $g=g(a, \xi)$ and $\mathcal{L}=\mathcal{L}(a, \ldots)$ (remember $a \equiv u \bar{u})$, which holds in all cases we want to study, and get

$$
\begin{aligned}
\left.\partial^{\mu} J_{\mu}^{(Y)}\right|_{\mathrm{s}}= & \left(\bar{u} Z^{u}+u Z^{\bar{u}}\right)\left(h_{a} u^{\mu} \pi_{\mu}+h \mathcal{L}_{a}\right)+ \\
& Z^{\xi}\left(h_{\xi} u^{\mu} \pi_{\mu}+h \mathcal{L}_{\xi}\right)+ \\
& \left(h Z^{\xi}\right)_{\xi}\left(\xi^{\mu} P_{\mu}-u^{\mu} \pi_{\mu}\right) .
\end{aligned}
$$

If we do not assume any restriction on the Lagrangian, this expression is zero provided that $Z^{\xi}=0$ and $\left(\bar{u} Z^{u}+u Z^{\bar{u}}\right)=0$. The latter equation has the general solution $Z^{u}=i G_{\bar{u}}=i u G_{a}, Z^{\bar{u}}=-i G_{u}=-i \bar{u} G_{a}$ where $G(a, \xi)$ is an arbitrary function of its arguments. In short, the set of conserved currents in the strong integrability subsector and for general Lagrangian is given by the vector fields

$Y^{u}=i h G_{\bar{u}}=i h u G_{a}, \quad Y^{\bar{u}}=-i h G_{u}=-i h \bar{u} G_{a}, \quad Y^{\xi}=0, \quad G=G(a, \xi)$.

In terms of the Clebsch variables $A, B$, this set is given by $A=G(a, \xi)$, $B=\xi$, so it is precisely equal to the abelian subalgebra of Eq. (54).

Next, we want to restrict the possible Lagrangians so that the currents remain conserved for a larger class of $Y^{i}$. The first term, Eq. (81), is zero provided that

$$
\left(h_{a} u^{\mu} \pi_{\mu}+h \mathcal{L}_{a}\right)=0
$$

or, more explicitly,

$$
h_{a}\left(b \mathcal{L}_{b}+2 c \mathcal{L}_{c}+e \mathcal{L}_{e}\right)+h \mathcal{L}_{a}=0 .
$$

This linear first order PDE may be easily solved by the method of characteristics and has the general solution

$$
\mathcal{L}(a, b, c, \xi, d, e)=\mathcal{F}\left(\frac{b}{h}, \frac{c}{h^{2}}, \xi, d, \frac{e}{h}\right)
$$

where $\mathcal{F}$ is an arbitrary function of its arguments. Therefore, Eq. (866) fixes the dependence on $a$ in terms of the dependence on $h$ (i.e., $g=h^{-1}$ ). Eq. (82) is zero if

$$
\left(h_{\xi} u^{\mu} \pi_{\mu}+h \mathcal{L}_{\xi}\right)=0,
$$


or

$$
h_{\xi}\left(b \mathcal{L}_{b}+2 c \mathcal{L}_{c}+e \mathcal{L}_{e}\right)+h \mathcal{L}_{\xi}=0
$$

with the general solution

$$
\mathcal{L}(a, b, c, \xi, d, e)=\mathcal{F}\left(a, \frac{b}{h}, \frac{c}{h^{2}}, d, \frac{e}{h}\right) .
$$

A general solution to both equations is, therefore,

$$
\mathcal{L}(a, b, c, \xi, d, e)=\mathcal{F}\left(\frac{b}{h}, \frac{c}{h^{2}}, d, \frac{e}{h}\right)
$$

and fixes the dependence both on $a$ and on $\xi$ in terms of a dependence on $h$. The Skyrme model is in this class with

$$
g=h^{-1}=4 \frac{\sin ^{2} \xi}{(1+a)^{2}} .
$$

Finally, expression ( 83 ) is zero if the Lagrangian obeys

$$
\left(\xi^{\mu} P_{\mu}-u^{\mu} \pi_{\mu}\right)=0
$$

or, more explicitly,

$$
b \mathcal{L}_{b}+2 c \mathcal{L}_{c}-2 d \mathcal{L}_{d}-e \mathcal{L}_{e}=0
$$

There exist Lagrangians which obey this additional condition, which may contain terms like, e.g., $(b / h)^{2} d$ or $\left(c / h^{2}\right) d$, etc. In fact, if we introduce a "weight number" which associates weight +1 with each power of the derivative $u_{\mu}$ and $\bar{u}_{\mu}$, and a weight -2 with each power of the derivative $\xi_{\mu}$, then Eq. (94) just requires that the total weight of each term in the Lagrangian is zero, i.e.

$$
\mathcal{W} \equiv \operatorname{pow}\left(u_{\mu}\right)+\operatorname{pow}\left(\bar{u}_{\mu}\right)-2 \operatorname{pow}\left(\xi_{\mu}\right)=0
$$

(where e.g., $\mathcal{W}(b)=2$ or $\mathcal{W}(e)=-2$ or $\mathcal{W}(d)=-4$ ).

The Skyrme Lagrangian, however, does not obey Eq. (94). Therefore, in this case Expression (

$$
\left(h Z^{\xi}\right)_{\xi} \equiv Y_{\xi}^{\xi} \equiv \partial_{3} Y^{3}=0,
$$

that is, exactly condition (48) of the last section. Therefore, a general Noether current $J_{\mu}^{(y)}$ is conserved in the strong integrability subsector of the Skyrme model provided that $Y$ obeys Eq. (96)). The currents (34), (37) and (38) belong to this class, but there may exist more generators $Y$ obeying condition (96). 
Next, let us relax the strong integrability conditions. We still require that

$$
u^{\mu} \xi_{\mu}=0
$$

but now $u_{\mu}^{2}$ need not be zero. This implies

$$
u^{\mu} \bar{\pi}_{\mu}=u_{\mu}^{2} \mathcal{L}_{b}, \quad u^{\mu} P_{\mu}=0, \quad \xi^{\mu} \pi_{\mu}=0
$$

and leads to the current divergence

$$
\left.\partial^{\mu} J_{\mu}^{(Y)}\right|_{\mathrm{w}}=\left.\partial^{\mu} J_{\mu}^{(Y)}\right|_{\mathrm{s}}+\left(Y_{\bar{u}}^{u} \bar{u}_{\mu}^{2}+Y_{u}^{\bar{u}} u_{\mu}^{2}\right) \mathcal{L}_{b} .
$$

This divergence is zero provided that, in addition to $\left.\partial^{\mu} J_{\mu}^{(Y)}\right|_{\mathrm{S}}=0$, either $\mathcal{L}_{b}=0$, i.e., the Lagrangian does not depend on $b$, or $Y$ is again restricted to the abelian subalgebra (84). Then the above divergence can be reexpressed like

$$
\left.\partial^{\mu} J_{\mu}^{(Y)}\right|_{\mathrm{w}}=\left.\partial^{\mu} J_{\mu}^{(Y)}\right|_{\mathrm{s}}+\left[\partial_{a}\left(h G_{a}\right)\right]\left(u^{2} \bar{u}_{\mu}^{2}-\bar{u}^{2} u_{\mu}^{2}\right) \mathcal{L}_{b}
$$

and can be made zero by imposing the weak integrability condition

$$
u^{2} \bar{u}_{\mu}^{2}-\bar{u}^{2} u_{\mu}^{2}=0
$$

in addition to $u^{\mu} \xi_{\mu}=0$. Therefore, the currents $J_{\mu}^{(Y)}$ are conserved for $Y$ belonging to the abelian subalgeba (84) if the weak integrability conditions

$$
u^{2} \bar{u}_{\mu}^{2}-\bar{u}^{2} u_{\mu}^{2}=0, \quad u^{\mu} \xi_{\mu}=0
$$

are fulfilled. This is true for arbitrary Lagrangians, because $\left.\partial^{\mu} J_{\mu}^{(Y)}\right|_{\mathrm{s}}$ is identically zero for $Y$ belonging to the abelian subalgebra (84). Therefore, these conserved currents exist in the weak integrable subsector of the Skyrme model.

The weak integrability conditions (102) have a nice geometrical interpretation in terms of the real functions $(\xi, \Sigma, \Lambda)$ where $u=\exp (\Sigma+i \Lambda)$, see ([24]). They are then equivalent to the perpendicularity conditions

$$
\xi^{\mu} \Sigma_{\mu}=0, \quad \xi^{\mu} \Lambda_{\mu}=0, \quad \Sigma^{\mu} \Lambda_{\mu}=0 .
$$

Specifically, for time-independent field configurations these are just the conditions that $(\xi, \Sigma, \Lambda)$ form a set of perpendicular curvilinear coordinates in base space $\mathbb{R}^{3}$.

Finally, let us calculate the full divergence. We get

$$
\begin{aligned}
\partial^{\mu} J_{\mu}^{(Y)}= & \left.\partial^{\mu} J_{\mu}^{(Y)}\right|_{\mathrm{w}}+\left\{\left(u^{\mu} \xi_{\mu}\right)^{2} \mathcal{L}_{e} Y_{u}^{\bar{u}}+\right. \\
& u^{\mu} \xi_{\mu}\left[\left(\mathcal{L}_{b}+2 b \mathcal{L}_{c}+d \mathcal{L}_{e}\right) Y_{\xi}^{\bar{u}}+\left(2 \mathcal{L}_{d}+b \mathcal{L}_{e}\right) Y_{u}^{\xi}\right] \\
& \left.+u_{\nu}^{2} \bar{u}^{\mu} \xi_{\mu}\left(2 \mathcal{L}_{c} Y_{\xi}^{\bar{u}}-\mathcal{L}_{e} Y_{u}^{\xi}\right)+\text { h.c. }\right\}
\end{aligned}
$$


which can be made equal to zero in different ways.

If no constraints (integrability conditions) are imposed, obviously only the Noether currents of the target space symmetries remain. For a completely generic Lagrangian the only remaining conserved current is the one with $Y$ given by

$$
Y^{u}=i u, \quad Y^{\bar{u}}=-i \bar{u}, \quad Y^{\xi}=0
$$

which corresponds to the symmetry $u \rightarrow e^{i \alpha} u$ and is always present for Lagrangians $\mathcal{L}=\mathcal{L}(a, b, c, \xi, d, e)$ by construction. If the Lagrangian obeys the condition (91), like is the case, e.g., in the Skyrme model, then still there exist only finitely many conserved currents and, moreover, the corresponding charges are now the Noether charges of the isometries of the (target space) metric

$$
d s^{2}=d \xi^{2}+g d u d \bar{u}
$$

But there also exist Lagrangians with infinitely many symmetries. Indeed, let us restrict to a subalgebra of the abelian subalgebra (84) such that $\partial_{\xi} Y^{u}=0$ in addition to $Y^{\xi}=0$. This is achieved by setting

$$
Y^{u}=i u \tilde{G}_{a}, \quad Y^{\bar{u}}=-i \bar{u} \tilde{G}_{a}, \quad Y^{\xi}=0, \quad \tilde{G}=\tilde{G}(a), \quad \tilde{G}_{\xi} \equiv 0 .
$$

These vector fields obey $\partial_{i}\left(g Y^{i}\right)=\partial_{u}\left(g Y^{u}\right)+\partial_{\bar{u}}\left(g Y^{\bar{u}}\right)=0$ for $g=g(a, \xi)$, as may be checked easily, and are, therefore, volume preserving diffeomorphisms forming a subalgebra of the abelian subalgebra (844). In the above current divergence, the second and third line (Eqs. (105) and (106)) are zero for $Y$ of this type, and only the first line, Eq. (104), remains. The second term of the first line vanishes if we assume $\mathcal{L}_{e}=0$, i.e., $\mathcal{L}=\mathcal{L}(a, b, c, \xi, d)$. Further, $\left.J_{\mu}^{(Y)}\right|_{\mathrm{s}}$ is automatically conserved. It remains to investigate the additional term in $\left.J_{\mu}^{(Y)}\right|_{\mathrm{w}}$. For $\mathcal{L}_{b}=0$ this additional term is zero, and it follows that a field theory with a Lagrangian $\mathcal{L}(a, c, \xi, d)$ has infinitely many conserved currents $J_{\mu}^{(Y)}$ with $Y$ given by (109) for the full model (i.e., without additional integrability conditions). It has, therefore, infinitely many target space Noether symmetries.

Alternatively, an arbitrary model $\mathcal{L}(a, b, c, \xi, d)$ has infinitely many conserved currents $J_{\mu}^{(Y)}$ with $Y$ given by (109) in the submodel defined by the weak integrability condition (101).

As we have seen, there are quite many possibilities for having conserved currents for different models (i.e., different Lagrangians) or their submodels defined by some additional integrability conditions. Therefore, we summarize our results in the Tables $1-3$. Observe that there exist infinitely many conserved currents in all cases of Table 1 and Table 2 . In Table 3 there are 
only finitely many conserved currents in case a) and b), whereas there exist infinitely many conservation laws in the remaining cases.

The Skyrme model without the pion mass term corresponds to case b) of Table 1 (for the strong integrability conditions (76)), to case a) of Table 2 (for the weak integrability conditions (102), or to case b) of Table 3 (the isometries of the target space $S^{3}$ ). The Skyrme model with pion mass term corresponds to case a) of Table 1 and to case a) of Table 2, that is, it has the same conserved currents for the strong and for the weak intergrability conditions. Further, it corresponds to case a) of Table 3 where it has, however, three conserved currents (corresponding to three target space symmetries) rather than just one in the most generic case (i.e., the Skyrme model Lagrangian with pion mass term is still somewhat "special" and has an enhanced symmetry).

Recently, another class of models has been studied, and infinitely many soliton solutions have been found analytically (see Ref. [24]), for the class of Lagrangians

$$
\mathcal{L}=-f^{(1)}(a) f^{(2)}(\xi) c^{\frac{3}{4}}+d^{\frac{3}{2}},
$$

where the non-integer power of the kinetic terms has been chosen carefully in order to avoid the Derrick scaling argument against the existence of soliton solutions. Further, $f^{(1)}$ and $f^{(2)}$ are arbitrary functions of their arguments. Lagrangians of this type belong to case c) of Table 3 and have, therefore, infinitely many target space symmetries and infinitely many conserved charges.

\section{Conclusions}

We have used geometric structures of the target space to find and classify the conservation laws in a large class of Skyrme type models. More precisely, we have analysed under which conditions there exist infinitely many conservation laws either in the full theories or in submodels defined by additional integrability conditions. It turned out that the conserved charges belong to certain subsets of the Noether charges of volume-preserving target space diffeomorphisms in all cases. These conservation laws should be helpful for the further study of these models, e.g., for finding solutions, either exact or numerical. In more general terms, the integrability conditions could serve as an alternative to the BPS conditions, which are missing in the Skyrme model - in analogy to the $\mathrm{CP}^{n}$ models, where the strong integrable subsector is precisely equivalent to the BPS sector, whereas the weak integrable subsector also allows for non-BPS solutions, see [24].

Specifically, for the Skyrme model we were able to re-derive the results of [15] and of [24] on the strong and weak integrable subsectors of the Skyrme 


\begin{tabular}{|ll|}
\hline & Integrability conditions $u_{\mu}^{2}=0$ and $u^{\mu} \xi_{\mu}=0$. \\
\hline a) & no condition on $\mathcal{L}$. \\
& $Y$ forms the abelian subalgebra $($ for $G=G(a, \xi))$ : \\
& $Y^{u}=i h u G_{a}, \quad Y^{\bar{u}}=-i h \bar{u} G_{a}, \quad Y^{\xi}=0$. \\
\hline b) & $\mathcal{L}=\mathcal{F}\left(\frac{b}{h}, \frac{c}{h^{2}}, d, \frac{e}{h}\right)$, see Eq. (91) \\
& $Y$ form the subset $Y_{\xi}^{\xi}=0$ (is not a subalgebra). \\
\hline c) & $\mathcal{W}(\mathcal{L})=0$, see Eq. (95) $).$ \\
& no further condition on $Y$. \\
\hline
\end{tabular}

Table 1: Conserved currents $J_{\mu}^{(Y)}$ for the strong integrability conditions.

The vector fields $Y$ always generate volume-preserving diffeomorphisms, i.e., they obey $\partial_{i}\left(g Y^{i}\right)=0$.

A general Lagrangian is of the form $\mathcal{L}=\mathcal{L}(a, b, c, \xi, d, e)$.

theory, and to put these results into a more geometric context. All the conserved currents in the strong integrable subsector of the Skyrme model found in [15] belong to the subset of the volume preserving diffeomorphisms obeying $\partial_{3} Y^{3}=0$, see e.g. case b) in Table 1 . It is however possible that this set is not exhausted by the currents given explicitly in [15] (see Eqs. (34), (37), (38)), that is, there might exist more currents in this subset. Interestingly, this subset does not form a subalgebra. The conserved charges in the weak integrable subsector of the Skyrme model found in [24] form an abelian subalgebra of the Lie algebra of volume preserving target space diffeomorphisms, which is given e.g. in case a) of Table 2. The fact that the conserved charges do form a Lie subalgebra for the weak integrability conditions but not for the strong integrability conditions makes the former ones appear somewhat more natural.

Further, the results of [22 for a two-dimensional target space may be recovered easily from the results presented here by simply assuming that nothing depends on the third target space coordinate $X^{3} \equiv \xi$ and by setting equal to zero the corresponding vector component, i.e., $Y^{3}=0$. Finally, let us remark that our results could also shed more light on the original zero curvature construction of [1, and might, for instance, help in finding the appropiate 2 form or even more general connections for this construction. This problem is under investiagtion.

\section{Acknowledgements}

C. A. and J.S.-G. thank MCyT (Spain) and FEDER (FPA2005-01963), and Incentivos from Xunta de Galicia. A.W. gratefully acknowledges Departa- 


\begin{tabular}{|ll|}
\hline \multicolumn{1}{|c|}{ Integrability conditions $u^{\mu} \xi_{\mu}=0}$. \\
\hline a) & no condition on $\mathcal{L} ;$ or $\mathcal{L}=\mathcal{F}\left(\frac{b}{h}, \frac{c}{h^{2}}, d, \frac{e}{h}\right)$. \\
& $Y$ forms the abelian subalgebra $($ for $G=G(a, \xi)):$ \\
& $Y^{u}=i h u G_{a}, \quad Y^{\bar{u}}=-i h \bar{u} G_{a}, \quad Y^{\xi}=0$. \\
& And the further integrability condition $u^{2} \bar{u}_{\mu}^{2}-\bar{u}^{2} u_{\mu}^{2}$ holds. \\
\hline b) & $\mathcal{L}_{b}=0$. \\
& $Y$ forms the abelian subalgebra $($ for $G=G(a, \xi)):$ \\
& $Y^{u}=i h u G_{a}, \quad Y^{\bar{u}}=-i h \bar{u} G_{a}, \quad Y^{\xi}=0$. \\
\hline c) & $\mathcal{L}_{b}=0$ and $\mathcal{L}=\mathcal{F}\left(\frac{b}{h}, \frac{c}{h^{2}}, d, \frac{e}{h}\right)$. \\
& $Y$ form the subset $Y_{\xi}^{\xi}=0($ is not a subalgebra $)$. \\
\hline d) & $\mathcal{L}_{b}=0$ and $\mathcal{L}=\mathcal{F}\left(\frac{b}{h}, \frac{c}{h^{2}}, d, \frac{e}{h}\right)$ and $\mathcal{W}(\mathcal{L})=0$. \\
& no further condition on $Y$. \\
\hline
\end{tabular}

Table 2: Conserved currents $J_{\mu}^{(Y)}$ for the weak integrability conditions.

The vector fields $Y$ always generate volume-preserving diffeomorphisms, i.e., they obey $\partial_{i}\left(g Y^{i}\right)=0$.

A general Lagrangian is of the form $\mathcal{L}=\mathcal{L}(a, b, c, \xi, d, e)$.

\begin{tabular}{|l|l|}
\hline \multicolumn{1}{|c|}{ No integrability conditions. } \\
\hline a) & no condition on $\mathcal{L}$. \\
& Generically there exists only one vector field $Y$ : \\
& $Y^{u}=i u, \quad Y^{\bar{u}}=-i \bar{u}, \quad Y^{\xi}=0$. \\
\hline b) & $\mathcal{L}=\mathcal{F}\left(\frac{b}{h}, \frac{c}{h^{2}}, d, \frac{e}{h}\right)$. \\
& There exist finitely many $Y$ generating the \\
& target space isometries for the metric of Eq. (108). \\
\hline c) & $\mathcal{L}_{b}=0$ and $\mathcal{L}_{e}=0$. \\
& $Y$ form the abelian subalgebra (see Eq. (109), where $\tilde{G}=\tilde{G}(a))$ : \\
& $Y^{u}=i u \tilde{G}_{a}, \quad Y^{\bar{u}}=-i \bar{u} \tilde{G}_{a}, \quad Y^{\xi}=0$. \\
\hline d) & $\mathcal{L}_{b}=0$ and $\mathcal{L}_{e}=0$. \\
& $Y$ forms the abelian subalgebra (for $G=G(a, \xi)):$ \\
& $Y^{u}=i h u G_{a}, \quad Y^{\bar{u}}=-i h \bar{u} G_{a}, \quad Y^{\xi}=0$. \\
& $A n d$ the further integrability condition $u^{2} \bar{u}_{\mu}^{2}-\bar{u}^{2} u_{\mu}^{2}$ holds. \\
\hline e) & $\mathcal{L}_{e}=0$. \\
& $Y$ form the abelian subalgebra (see Eq. (109), where $\tilde{G}=\tilde{G}(a))$ : \\
& $Y^{u}=i u \tilde{G}_{a}, \quad Y^{\bar{u}}=-i \bar{u} \tilde{G}_{a}, \quad Y^{\xi}=0$. \\
& $A n d$ the further integrability condition $u^{2} \bar{u}_{\mu}^{2}-\bar{u}^{2} u_{\mu}^{2}$ holds. \\
\hline
\end{tabular}

Table 3: Conserved currents $J_{\mu}^{(Y)}$ without further integrability conditions. The vector fields $Y$ always generate volume-preserving diffeomorphisms, i.e., they obey $\partial_{i}\left(g Y^{i}\right)=0$.

A general Lagrangian is of the form $\mathcal{L}=\mathcal{L}(a, b, c, \xi, d, e)$. 
mento de Fisica de Particulas, Universidad de Santiago for hospitality. Further, C. A. acknowledges support from the Austrian START award project FWF-Y-137-TEC and from the FWF project P161 05 NO 5 of N.J. Mauser.

\section{References}

[1] O. Alvarez, L.A. Ferreira, and J. Sánchez-Guillén, Nucl. Phys. B 529, 689 (1998).

[2] H. Aratyn, L.A. Ferreira, and A. Zimerman, Phys. Lett. B456 (1999) 162.

[3] H. Aratyn, L.A. Ferreira, and A. Zimerman, Phys. Rev. Lett. 83 (1999) 1723.

[4] E. De Carli, L.A. Ferreira, J. Math. Phys. 46 (2005) 012703; hep-th/0406244.

[5] A. Wereszczynski, Eur. Phys. J. C38 (2004) 261. e-Print Archive: hep-th/0405155.

[6] L.A. Ferreira, JHEP 0603 (2006) 075; hep-th/0601235.

[7] A.C. Riserio do Bonfim, L.A. Ferreira, JHEP 0603 (2006) 097; hep-th/0602234.

[8] D. A. Nicole, J. Phys. G4 (1978) 1363.

[9] A. Wereszczynski, Eur. Phys. J. C41 (2005) 265; math-ph/0504008.

[10] A. Wereszczynski, Phys. Lett. B621 (2005) 201, hep-th/0508121.

[11] C. Adam, J. Sánchez-Guillén, R.A. Vázquez, and A. Wereszczyński, J. Math. Phys. 47, 052302 (2006), hep-th/0602152.

[12] R. S. Ward, Nonlinearity 12 (1999) 241.

[13] C. Adam, J. Sanchez-Guillen, A. Wereszczynski, Eur. Phys. J. C47 (2006) 513; hep-th/0602008.

[14] T.H.R. Skyrme, Proc. R. Soc. Lond. A260 (1961) 127; Nucl. Phys. 31 (1962) 556.

[15] L.A. Ferreira, J. Sanchez Guillen, Phys. Lett. B504 (2001) 195; hep-th/0010168. 
[16] A. Gustavsson, hep-th/0608141.

[17] U. Schreiber, PhD Thesis, hep-th/0509163.

[18] A. Lahiri, hep-th/0402227.

[19] R. Attal, math-ph/0205017.

[20] O. Babelon and L.A. Ferreira, JHEP 0211 (2002) 020.

[21] C. Adam, J. Sanchez-Guillen, Phys. Lett. B626 (2005) 235; hep-th/0508011.

[22] C. Adam, J. Sanchez-Guillen, A. Wereszczynski, J. Math. Phys. 47 (2006) 022303; hep-th/0511277.

[23] J. Sanchez-Guillen, Phys. Lett. B548 (2002) 252, Erratum-ibid. B550 (2002) 220; hep-th/0208215.

[24] C. Adam, J. Sanchez-Guillen, A. Wereszczynski, hep-th/0610024.

[25] N.S. Manton, Commun. Math. Phys. 111 (1987) 469.

[26] V.G. Makhankov, Y.P. Rubakov, and V.J. Sanyuk, "The Skyrme Model", Springer Verlag, Berlin 1993.

[27] T. Gisiger, Manu B. Paranjape, Phys. Rept. 306 (1998) 109; hep-th/9812148.

[28] N. Manton, P. Sutcliffe, "Topological Solitons", Cambridge University Press, Cambridge, 2004. 